\title{
Correlation research between fear of disease progression and quality of life in patients with lung cancer
}

\author{
Xiaofeng Kuang ${ }^{1 \#}$, Fengjiao Long ${ }^{1 \#}$, Hui Chen ${ }^{1}$, Ying Huang ${ }^{1}$, Lanman He ${ }^{1}$, Lin Chen ${ }^{1}$, Lipin Xie $^{1}$, \\ Jinxiao $\mathrm{Li}^{1}$, Yuanling Luo ${ }^{1}$, Hongmei Tao ${ }^{2}$ \\ ${ }^{1}$ Department of Oncology, Cancer Center of the Fifth Affiliated Hospital of Sun Yat-sen University, Zhuhai, China; ${ }^{2}$ Department of Medicine, The \\ Fifth Affiliated Hospital of Sun Yat-sen University, Zhuhai, China \\ Contributions: (I) Conception and design: All authors; (II) Administrative support: All authors; (III) Provision of study materials or patients: All \\ authors; (IV) Collection and assembly of data: All authors; (V) Data analysis and interpretation: All authors; (VI) Manuscript writing: All authors; (VII) \\ Final approval of manuscript: All authors. \\ \#These authors contributed equally to this work. \\ Correspondence to: Yuanling Luo. Department of Oncology, Cancer Center of the Fifth Affiliated Hospital of Sun Yat-sen University, 52 East Meihua \\ Road, Zhuhai 519000, China. Email: 982757953@qq.com; Hongmei Tao. Department of Medicine, The Fifth Affiliated Hospital of Sun Yat-sen \\ University, 52 East Meihua Road, Zhuhai 519000, China. Email: 406045976@qq.com.
}

\begin{abstract}
Background Lung cancer has the highest incidence rate and mortality rate of all malignancies. In recent years, the therapeutic effect of lung cancer has been greatly improved, but the fear of disease progression still directly affects the quality of life (QOL) of patients. The aim of this study was to evaluate the factors affecting the progression of fear of disease and its impact on the quality of life in patients with lung cancer.

Methods: From December 2019 to February 2020, 102 patients with lung cancer in the Department of Thoracic Oncology of a top three hospital were investigated by using the simplified fear of disease progression scale (FoP-Q-SF) and the quality-of-life scale for cancer patients (FACT-G). Data were collected and statistically analyzed by SPSS25.0 software.
\end{abstract}

Results: A total of 110 questionnaires were distributed and 102 valid questionnaires were recovered, indicating a recovery rate of $92.7 \%$. The results of multiple stepwise regression analyses showed that blood group, monthly income, and mood state were the influencing factors for the progression of phobic diseases in cancer patients $(\mathrm{P}<0.05)$, and the score of progression of phobic disease was negatively correlated with the quality-of-life score $(\mathrm{r}=-0.382)$.

Conclusions: The progress of phobic diseases in patients with lung cancer seriously affects their QOL, and further attention by medical staff in providing health education, psychological counseling, social support, and other measures is required.

Keywords: Lung cancer; fear of disease progression; quality of life (QOL)

Submitted Sep 13, 2021. Accepted for publication Nov 03, 2021.

doi: 10.21037/apm-21-2821

View this article at: https://dx.doi.org/10.21037/apm-21-2821

\section{Introduction}

Cancer has become the second leading cause of mortality, with nearly $1 / 6$ of deaths worldwide caused by the disease (1). Lung cancer has the highest incidence rate among cancer patients in China (2), comprising close to $20 \%$ of all malignancies (3). Fear of disease progression, or "fear of progress" (FoP) (4) in cancer, is a psychological state in which patients are afraid of the possibility of metastasis or recurrence of the disease. One study showed that the FoP score of breast cancer patients in China is mostly moderate (5). Poor quality of life (QOL) is a common problem in all cancer patients, and this problem is more prominent in lung cancer, mainly including physical, social, emotional, 
and functional status. It has become a vital consideration in understanding the long-term impact of cancer and its treatment (6), and studies have shown that FoP has a direct impact on QOL in people with interstitial lung disease and prostate cancer patients $(7,8)$. Due to the FoP, patients will have large emotional fluctuations, which will eventually affect the treatment effect and quality of life. At present, there are few studies on FoP in lung cancer patients in China (9), and in this study we hoped to develop an understanding of its relationship with QOL in lung cancer patients to increase awareness in medical settings and the community. We present the following article in accordance with the SURGE reporting checklist (available at https://apm.amegroups.com/ article/view/10.21037/apm-21-2821/rc).

\section{Methods}

\section{Study population}

A questionnaire survey was conducted on 110 patients with primary lung cancer in the Thoracic Oncology Department of the Fifth Affiliated Hospital of Sun Yat-sen University from December 2019 to January 2020. The inclusion criteria were: (I) age $>18$ years old, with self-judgment ability; (II) patients were hospitalized for $\geq 1$ day; (III) hospitalized patients with a pathological diagnosis of lung cancer; (IV) patients were conscious, had basic language communication skills, and had the ability to complete the questionnaire survey; (V) patients had a high degree of cooperation and voluntarily accepted the survey and informed consent of their families. The exclusion criteria were: (I) patient's age was less than 18 years old; (II) patients were unconscious and unable to complete the questionnaire; (III) patients were hospitalized repeatedly.

\section{Study methods}

The sampling method was used in this study. Comprehensive quality control measures have been taken in the whole process of investigation (including design, investigation and analysis stages), such as rationalization and standardization of problem establishment, pre investigation, and timely study and solve the problems in the investigation. Trained investigators were selected to conduct clinical investigations using a unified questionnaire. All procedures performed in this study involving human participants were in accordance with the Declaration of Helsinki (as revised in 2013). The study was approved by ethics board of the Fifth Affiliated
Hospital of Sun Yat-sen University (K247-1) and written informed consent was obtained from all patients.

\section{Patient general information questionnaire}

The questionnaire included the general information of patients, as well as blood type, occupation, monthly income, medical burden, place of residence, religious belief, main caregivers, family companionship, mood state, efforts for serious illness, and the decision maker for existing treatment plans.

\section{Simplified Chinese scale for fear of disease progression in cancer patients (FoP-Q-SF)}

The scale was developed by Mehnert of Germany on the basis of FoP-Q in 2006 (10), and the simplified Chinese version of the scale (FoP-Q-SF) adopts a Likert 5-point scoring method, with a total of 11 items (11). The evaluation method adopts patient self-evaluation, with a score of 11-55 points, and the higher the patient's score, the stronger their fear of disease progression. The Internal consistency Cronbach's $\alpha$ coefficient is 0.883 in the original FoP-Q-SF total table, and the test-retest reliability was 0.85 after modifying the original scale. The FoP-Q-SF has good reliability and validity and is suitable for the detection of disease progression fear in lung cancer patients.

\section{QOL scale for cancer patients (FACT-G)}

This is a specific scale for various tumor patients formed by combining common and specific templates and is especially used to measure the QOL. The scale is mainly divided into four dimensions: physiological, social, emotional, and functional status, with a total of 23 items (12). In this study, a Likert scoring method was adopted for all items of the FACT-G, most of which were positive items, while reverse items were GP1-GP7, GE1, and GE3-GE6. The total score of patients ranged from high to low and indicated that the QOL of patients with lung cancer ranged from good to poor. The value reliability of internal consistency in each field in the original scale $\alpha$ is 0.85 , and the test-retest reliability was 0.902 after modifying the original scale. FACT-G has good reliability and validity, and is suitable for the detection of QOL in patients with lung cancer.

\section{Statistical analysis}

After the data were collected, SPSS25.0 statistical software was used for descriptive statistics, independent sample $t$-test, one-way analysis of variance (ANOVA), Pearson correlation analysis, and multiple linear regression in the data analysis. 


\section{Results}

\section{Patients}

A total of 110 questionnaires were distributed and 102 valid questionnaires were recovered, indicating a recovery rate of $92.7 \%$. Patients comprised of 45 females $(44.1 \%)$ and 57 males $(55.9 \%)$; there were 88 cases $(86.3 \%)$ of nonsmall cell carcinoma and 14 cases $(13.7 \%)$ of small cell carcinoma; and the age range was $25-79$ years old, of which those between 51-70 years old (47.1\%) accounted for most respondents. Most patients were married (97, 95.1\%), and their educational level was concentrated in high school and above (70, 68.6\%). See Table 1 for details.

\section{FoP in cancer patients}

Univariate analysis showed that there were statistically significant differences in the level of FoP among lung cancer patients in terms of their monthly income, blood type, medical insurance type, main caregivers, family companionship, and $\operatorname{mood}(\mathrm{P}<0.05)$, see Table 1 for details.

\section{Multiple stepwise regression analysis of FoP}

Taking the statistically significant factors obtained from the univariate analysis of $\mathrm{FoP}$ as the independent variable and the total score of FoP as the dependent variable, the FoP was analyzed by multiple stepwise regression. The results showed that the monthly income, mood state and blood type of lung cancer patients all affected the $\mathrm{FoP}(\mathrm{P}<0.01)$, and the total coefficient of certainty was 0.291 , indicating these three variables could explain $29.1 \%$ of the variation of the total FoP score. The assignment of independent variables of FoP and the results of multivariate analysis are shown in Tables 2,3.

\section{QOL in patients with lung cancer}

The total standardized QOL score of lung cancer patients was $56.15 \pm 21.69$. The standardized scores of the four dimensions of QOL were physiological dimension $(108.5 \pm 28.52)$, emotional dimension $(58.73 \pm 23.96)$, functional dimension $(57.11 \pm 25.15)$, and social/family dimension (32.95 \pm 30.21 ), see Table 4 .

\section{Progress of phobia in patients with lung cancer}

The FoP score of the 102 patients was $25.5 \pm 6.06$, which indicated a medium level. Among them, 18 patients had mild disease (17.65\%), 69 had moderate disease (67.65\%), and 15 had severe lung cancer (14.7\%). The top three items with the highest scores are shown in Table 5 .

\section{Correlation between fear, disease progression, and QOLI in patients with lung cancer}

The QOL score of lung cancer patients was significantly negatively correlated with the total score of FoP $(r=-0.382)$. Among them, the QOLI was positively correlated with the scores of social/family dimensions in the FoP ( $r=0.77)$, and negatively correlated with the scores of physiological dimensions $(r=-0.271)$, emotional dimensions $(r=-0.289)$, and functional dimensions $(r=-0.242)$ in the FoP, as shown in Table 6.

\section{Discussion}

A total of 102 questionnaires were collected in this study, of which the proportion of men and women was 57:45. Most patients were middle-aged and elderly, and more than $60 \%$ had intermediate and higher education. Most had non-small cell lung cancer, which were mostly advanced adenocarcinoma.

As We all know, anti-tumor treatment and drug side effects are inevitable. However, excessive worry, what we call fear of disease progression, will aggravate this subjective feeling of patients themselves. Finding out the objective factors affecting the progress of fear disease will help patients maintain a good attitude and accept everything calmly. The mood status of patients affected the FoP, with those in good mood and with a cheerful personality having a lower score and stronger tolerance for the disease (13). The monthly income of lung cancer patients also affected the FoP, with those with high income often receiving better and more timely treatment, and attracting a lower score. These findings show it is necessary for legislators to improve medical insurance and related policies for cancer patients to reduce the financial burden on patients (14).

The blood type of lung cancer patients also affected the FoP score. The results of this study showed that those with type $\mathrm{B}, \mathrm{AB}$, and $\mathrm{O}$ blood were more significantly afraid of disease progression than patients with type $A$. This result differs from those of previous studies and may be related to the controversial blood group theory (15), which suggests people with type $\mathrm{O}$ blood may have a lower risk of disease than other blood types.

In this study, while most patients experienced FoP, 
Table 1 Univariate analysis results of fear disease progression score of patients' general information ( $\mathrm{n}=102)$

\begin{tabular}{|c|c|c|c|c|c|}
\hline Characteristics & Cases (n) & Percentage (\%) & Fear disease progression score & $\mathrm{F} / \mathrm{t}$ & $P$ \\
\hline Male & 57 & 55.9 & $25.18 \pm 5.49$ & & \\
\hline Female & 45 & 44.1 & $25.91 \pm 6.75$ & & \\
\hline Age (years) & & & & 0.87 & 0.46 \\
\hline $31-55$ & 36 & 35.3 & $25.44 \pm 6.09$ & & \\
\hline $56-70$ & 48 & 47.1 & $26.13 \pm 5.96$ & & \\
\hline$>70$ & 15 & 14.7 & $23.33 \pm 6.34$ & & \\
\hline Medical insurance & & & & -0.08 & 0.93 \\
\hline Pleural effusion & & & & 1.61 & 0.11 \\
\hline Yes & 6 & 5.9 & $29.33 \pm 6.12$ & & \\
\hline No & 96 & 94.1 & $25.26 \pm 6.00$ & & \\
\hline Marriage & & & & 0.35 & 0.56 \\
\hline Yes & 5 & 4.9 & $25.43 \pm 6.11$ & & \\
\hline No & 97 & 95.1 & $25.28 \pm 4.79$ & & \\
\hline Degree of education & & & & 1.47 & 0.23 \\
\hline Junior high school and below & 18 & 17.6 & $24.89 \pm 6.05$ & & \\
\hline No & 20 & 19.6 & $24.8 \pm 7.67$ & & \\
\hline Radiotherapy & & & & 0.45 & 0.65 \\
\hline Yes & 34 & 33.3 & $25.88 \pm 5.51$ & & \\
\hline No & 68 & 66.8 & $25.31 \pm 6.34$ & & \\
\hline Targeted therapy & & & & -0.69 & 0.49 \\
\hline Yes & 42 & 41.2 & $25 \pm 6.74$ & & \\
\hline No & 60 & 58.8 & $25.85 \pm 5.56$ & & \\
\hline Immunotherapy & & & & -0.73 & 0.94 \\
\hline Yes & 13 & 12.7 & $25.38 \pm 4.03$ & & \\
\hline No & 89 & 87.3 & $25.52 \pm 6.31$ & & \\
\hline
\end{tabular}

Table 1 (continued) 
Table 1 (continued)

\begin{tabular}{|c|c|c|c|c|c|}
\hline Characteristics & Cases (n) & Percentage (\%) & Fear disease progression score & $\mathrm{F} / \mathrm{t}$ & $\mathrm{P}$ \\
\hline Blood type & & & & 4.51 & $0.005^{\star *}$ \\
\hline$A$ & 33 & 32.4 & $22.55 \pm 7.22$ & & \\
\hline $\mathrm{B}$ & 24 & 23.5 & $27.71 \pm 5.07$ & & \\
\hline$A B$ & 12 & 11.8 & $56.42 \pm 4.3$ & & \\
\hline $\mathrm{O}$ & 33 & 32.4 & $26.52 \pm 4.97$ & & \\
\hline$\leq 3,000$ & 34 & 33.3 & $28.26 \pm 5.8$ & & \\
\hline $3,000-5,000$ & 34 & 33.3 & $36.79 \pm 4.17$ & & \\
\hline $5,001-10,000$ & 27 & 26.5 & $20.93 \pm 6.21$ & & \\
\hline$\geq 10,000$ & 7 & 6.9 & $24.43 \pm 5.29$ & & \\
\hline Basically no burden & 14 & 13.7 & $23 \pm 9.22$ & & \\
\hline Have a certain burden & 42 & 41.2 & $26.55 \pm 5.01$ & & \\
\hline Have a heavy burden & 42 & 41.2 & $25.43 \pm 5.88$ & & \\
\hline Residence & & & & 0.05 & 0.96 \\
\hline City & 16 & 15.7 & $25.51 \pm 6.16$ & & \\
\hline Countryside & 86 & 84.3 & $25.44 \pm 5.67$ & & \\
\hline Occupation & & & & 1.82 & 0.17 \\
\hline Worker & 21 & 20.6 & $25.67 \pm 7.1$ & & \\
\hline Others & 34 & 33.3 & $26.79 \pm 4.67$ & & \\
\hline Religious belief & & & & 0.005 & 0.99 \\
\hline Yes & 4 & 3.9 & $25.75 \pm 3.10$ & & \\
\hline No & 98 & 96.1 & $25.49 \pm 6.16$ & & \\
\hline Primary caregiver & & & & 3.16 & $0.007^{\star \star}$ \\
\hline Mate & 73 & 71.6 & $25.27 \pm 5.74$ & & \\
\hline Children & 13 & 12.7 & $28.66 \pm 5.28$ & & \\
\hline Parents & 1 & 1.0 & 25 & & \\
\hline Brothers and sisters & 1 & 1.0 & 35 & & \\
\hline Medical staff & 9 & 8.8 & $26.67 \pm 5.77$ & & \\
\hline Escort & 1 & 1.0 & 19 & & \\
\hline Others & 4 & 3.9 & $16.25 \pm 6.19$ & & \\
\hline
\end{tabular}

Table 1 (continued) 
Table 1 (continued)

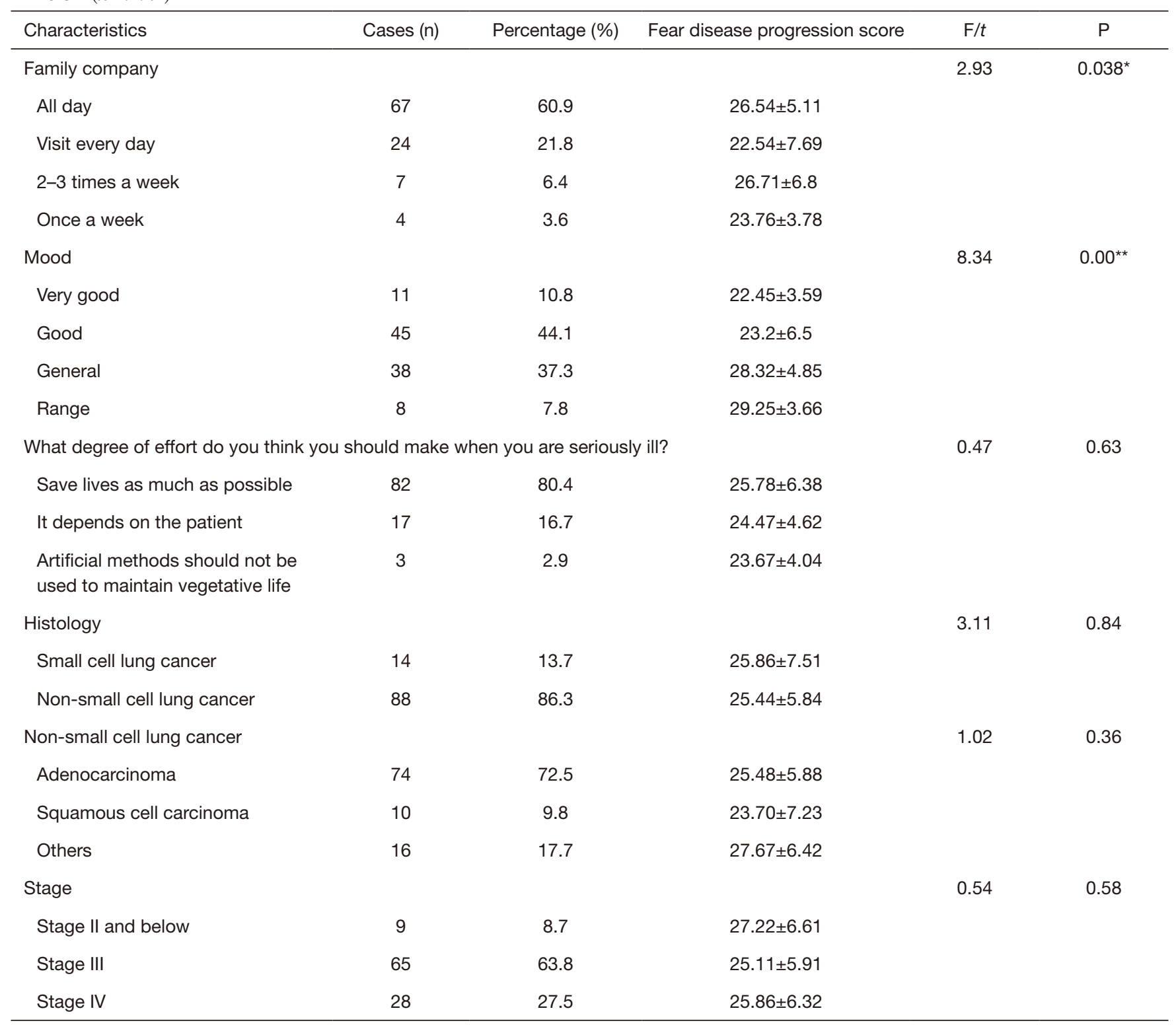

*, indicates $\mathrm{P}<0.05 ;{ }^{* *}$, indicates $\mathrm{P}<0.01$.

the score was $25.5 \pm 6.06$ points, which is considered a moderate level, and is basically consistent with the results of previous studies. In Table 5, the top three items with the highest scores in the questionnaire were the impact of the disease on patient's families, side effects of drugs, and work troubles. This may be because: (I) Chinese society generally has a strong concept of family, and not being a burden to family is important (16). At the same time, the complications or death caused by disease progression bring irreparable psychological and economic trauma to the families of patients (17). (II) Chemotherapy drugs used by cancer patients often cause adverse reactions (18), and bring further fear and potential resistance to the treatment. (III) Both illness and its treatment may disrupt the normal working lives of patients (19), and increase the economic burden of patients and their families (20).

Table 6 shows that the distribution of FoP in patients with lung cancer was significantly negatively correlated with the QOL score $(\mathrm{r}=-0.382, \mathrm{P}<0.05)$, indicating that the higher the level of FoP, the lower the QOL. The 
Table 2 Assignment of independent variable $(\mathrm{n}=102)$

\begin{tabular}{ll}
\hline Independent variable & Assignment method \\
\hline Did you receive immunotherapy & Yes $=1$, no $=2$ \\
Did you receive chemotherapy & Yes $=1$, no $=2$ \\
Medical burden & No burden at all $=1$, basically no burden $=2$, have a certain burden $=3$, have a heavy burden $=4$ \\
Blood type & $\mathrm{A}=1, \mathrm{~B}=2, \mathrm{AB}=3, \mathrm{O}=4$ \\
Occupation & Worker $=1$, farmer $=2$, cadre $=3$, medical staff $=4$, teacher $=5$, merchant $=6$, others $=7$ \\
Monthly income (Yuan) & $\leq 3,000=1,3,001-5,000=2,5,001-10,000=3, \geq 10,000=4$ \\
Primary caregiver & Mate $=1$, children $=2$, parents $=3$, brothers and sisters $=4$, medical staff $=5$, escort $=6$, others $=7$ \\
Family company & All day $=1$, visit every day $=2,2-3$ times a week $=3$, once a week $=4$ \\
Mood state & Very good $=1$, good $=2$, general $=3$, range $=4$
\end{tabular}

Table 3 Multiple stepwise regression analysis of fear of disease progression in cancer patients $(n=102)$

\begin{tabular}{|c|c|c|c|c|c|}
\hline Independent variable & \multicolumn{2}{|c|}{ Nonstandard coefficient } & Standardized partial regression coefficient & $T$ value & $P$ value \\
\hline Constant & 20.4 & 2.73 & - & 7.47 & $0.00^{\star *}$ \\
\hline Mood state & 2.701 & 0.678 & 0.352 & 3.986 & $0.00^{\star \star}$ \\
\hline Very good & - & & & & \\
\hline General & 5.861 & 1.879 & 0.47 & 3.119 & $0.002^{\star \star}$ \\
\hline Range & 6.795 & 2.55 & 0.303 & 2.665 & $0.009^{\star \star}$ \\
\hline Monthly income (Yuan) & -1.707 & 0.587 & -0.264 & -2.91 & $0.004^{\star *}$ \\
\hline$\leq 3,000$ & - & & & & \\
\hline$\geq 10,000$ & -3.63 & 2.239 & -0.152 & -1.621 & 0.108 \\
\hline \multicolumn{6}{|l|}{ Blood type } \\
\hline$A$ & - & & & & \\
\hline $\mathrm{B}$ & 5.163 & 1.546 & 0.363 & 3.339 & $0.001^{\star \star}$ \\
\hline $\mathrm{O}$ & 3.97 & 1.419 & 0.308 & 2.797 & $0.006^{* \star}$ \\
\hline$A B$ & 3.871 & 1.943 & 0.207 & 1.992 & $0.049^{\star}$ \\
\hline
\end{tabular}

Regression equation $R^{2}=0.291$, adjusted $R^{2}=0.269, f=13.382, P<0.01$. *, indicates $P<0.05$; ${ }^{*}$, indicates $P<0.01$.

FoP score was significantly negatively correlated with the scores of physiological dimension, emotional dimension, and functional dimension $(\mathrm{r}=-0.271,-0.289,-0.242$, $\mathrm{P}<0.05$ ), which mimic the results of other studies (21). This may be due to (I) the patients' lack of knowledge of cancer, doubts about the possibility of a cure, or fear of the adverse reactions of treatment. These adverse psychological emotions increased the psychological burden of patients and affect their QOL. (II) The emotional, social, and economic support of patients was difficult to 
Table 4 Quality of life scores of lung cancer patients ( $\mathrm{n}=102)$

\begin{tabular}{lccccc}
\hline Dimension & Score range & Minimum score & Maximum score & Original score, $\bar{x} \pm$ s & Standardized score, $\bar{x} \pm$ s \\
\hline Physiological condition & $0-28$ & 4 & 27 & $19.53 \pm 5.13$ & $108.5 \pm 28.52$ \\
Social/family status & $0-24$ & 0 & 18 & $11.58 \pm 6.94$ & $32.95 \pm 30.21$ \\
Emotional state & $0-16$ & 5 & 16 & $11.46 \pm 2.64$ & $58.73 \pm 23.96$ \\
Functional status & $0-24$ & 1 & 17 & $10.14 \pm 4.03$ & $57.11 \pm 25.15$ \\
Scale score (total) & $0-92$ & 28 & 72 & $52.71 \pm 9.54$ & $56.15 \pm 21.69$ \\
\hline
\end{tabular}

Table 5 Top three items with the highest scores of fear disease progression scale $(n=102)$

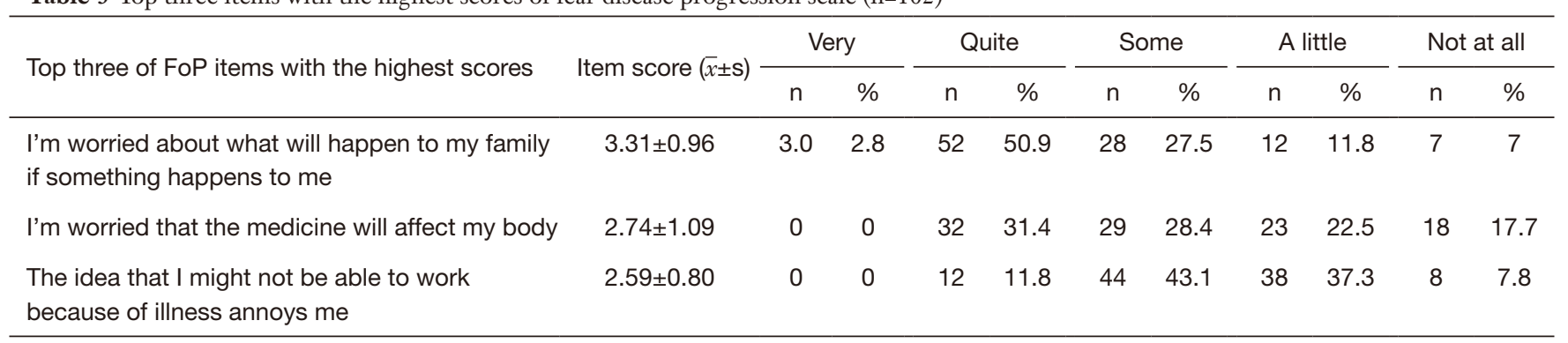

FoP, fear of progress.

Table 6 Correlation analysis between fear of disease progression and quality of life in patients with lung cancer $(n=102)$

\begin{tabular}{lcc}
\hline Dimension & $r$ & $P$ \\
\hline Physiological condition & -0.271 & $0.006^{\star *}$ \\
Social/family status & 0.77 & 0.443 \\
Emotional state & -0.289 & $0.003^{\star *}$ \\
Functional status & -0.242 & $0.014^{\star}$ \\
Scale score (total) & -0.382 & $0.000^{* *}$ \\
\hline
\end{tabular}

*, indicates $\mathrm{P}<0.05 ;{ }^{* *}$, indicates $\mathrm{P}<0.01$.

address, which may also affect their rehabilitation process. To address this, medical staff should take measures to increase the confidence of patients and to reduce FoP, such as reiterating the importance of family and social support to family members, providing ongoing counselling online and via telephone, and maintaining continuous care (22). The QOL score of patients was $(56.15 \pm 21.69)$, which was at the medium level (23). It can be seen from Table 4 that the physiological dimension score of patients with lung cancer was the highest $(108.5 \pm 28.52$ points), while that of society or family was the lowest $(32.95 \pm 30.21$ points), indicating that lung cancer caused considerable work, social, and family stress. In this study, following their illness, patients found it difficult to engage in their original work, resulting in reduced interpersonal communication and gradual alienation from society, which produced a variety of negative psychological emotions. Therefore, while using chemotherapy, radiotherapy, and other means to treat them, medical staff should attach great importance to the psychological status of patients (24), and encourage patients, families, and all involved parties to maintain close connections with those recovering from the disease.

In general, the fear of disease progression in patients with lung cancer seriously affects their QOL. With the continuous progress of medical treatment, physical disease rehabilitation is not the only factor medical workers should be concerned with, and the humanistic care of patients should be considered a crucial component of healing. Medical workers should pay more attention to patients' FoP and provide more health education, psychological counseling, social support, alleviate fear of disease progression for lung cancer patients to improve their QOL. This study has not investigated the impact of fear of disease recurrence on patients' QOL, and further research in this field is required.

\section{Acknowledgments}

Funding: None. 


\section{Footnote}

Reporting Checklist: The authors have completed the SURGE reporting checklist. Available at https://apm. amegroups.com/article/view/10.21037/apm-21-2821/rc

Data Sharing Statement: Available at https://apm.amegroups. com/article/view/10.21037/apm-21-2821/dss

Conflicts of Interest: All authors have completed the ICMJE uniform disclosure form (available at https://apm. amegroups.com/article/view/10.21037/apm-21-2821/coif). The authors have no conflicts of interest to declare.

Ethical Statement: The authors are accountable for all aspects of the work in ensuring that questions related to the accuracy or integrity of any part of the work are appropriately investigated and resolved. All procedures performed in this study involving human participants were in accordance with the Declaration of Helsinki (as revised in 2013). The study was approved by ethics board of the Fifth Affiliated Hospital of Sun Yat-sen University (K247-1) and written informed consent was obtained from all patients.

Open Access Statement: This is an Open Access article distributed in accordance with the Creative Commons Attribution-NonCommercial-NoDerivs 4.0 International License (CC BY-NC-ND 4.0), which permits the noncommercial replication and distribution of the article with the strict proviso that no changes or edits are made and the original work is properly cited (including links to both the formal publication through the relevant DOI and the license). See: https://creativecommons.org/licenses/by-nc-nd/4.0/.

\section{References}

1. Siegel RL, Miller KD, Jemal A. Cancer statistics, 2020. CA Cancer J Clin 2020;70:7-30.

2. Chen $W$, Zheng R, Baade PD, et al. Cancer statistics in China, 2015. CA Cancer J Clin 2016;66:115-32.

3. Gao S, Li N, Wang S, et al. Lung Cancer in People's Republic of China. J Thorac Oncol 2020;15:1567-76.

4. Lebel S, Ozakinci G, Humphris G, et al. Current state and future prospects of research on fear of cancer recurrence. Psychooncology 2017;26:424-7.

5. Niu L, Liang Y, Niu M. Factors influencing fear of cancer recurrence in patients with breast cancer: Evidence from a survey in Yancheng, China. J Obstet Gynaecol Res
2019;45:1319-27.

6. Firkins J, Hansen L, Driessnack M, et al. Quality of life in "chronic" cancer survivors: a meta-analysis. J Cancer Surviv 2020;14:504-17.

7. Haack M, Kramer S, Seidel G, et al. Quality of life and fear of disease progression are associated with aspects of health literacy in men with prostate cancer from Germany. Support Care Cancer 2020;28:2283-92.

8. Liu QW, Qin T, Hu B, et al. Relationship between illness perception, fear of progression and quality of life in interstitial lung disease patients: A cross-sectional study. J Clin Nurs 2021;30:3493-505.

9. Hu X, Wang W, Wang Y, et al. Fear of cancer recurrence in patients with multiple myeloma: Prevalence and predictors based on a family model analysis. Psychooncology 2021;30:176-84.

10. Mehnert A, Herschbach P, Berg P, et al. Fear of progression in breast cancer patients--validation of the short form of the Fear of Progression Questionnaire (FoP-Q-SF). Z Psychosom Med Psychother 2006;52:274-88.

11. Mahendran R, Liu J, Kuparasundram S, et al. Validation of the English and simplified Mandarin versions of the Fear of Progression Questionnaire - Short Form in Chinese cancer survivors. BMC Psychol 2020;8:10.

12. Bonomi AE, Cella DF, Hahn EA, et al. Multilingual translation of the Functional Assessment of Cancer Therapy (FACT) quality of life measurement system. Qual Life Res 1996;5:309-20.

13. Yang $\mathrm{Y}$, Sun $\mathrm{H}$, Liu T, et al. Factors associated with fear of progression in chinese cancer patients: sociodemographic, clinical and psychological variables. J Psychosom Res 2018;114:18-24.

14. Smith GL, Lopez-Olivo MA, Advani PG, et al. Financial Burdens of Cancer Treatment: A Systematic Review of Risk Factors and Outcomes. J Natl Compr Canc Netw 2019;17:1184-92.

15. Ewald DR, Sumner SC. Blood type biochemistry and human disease. Wiley Interdiscip Rev Syst Biol Med 2016;8:517-35.

16. Lee MC, Hinderer KA, Alexander CS. What Matters Most at the End-of-Life for Chinese Americans? Gerontol Geriatr Med 2018;4:2333721418778195.

17. Park HC, Kwon YE, Choi HY, et al. Health Insurance Status Is Related to Risk of Mortality and Hospitalization in Korean Maintenance Hemodialysis Patients: A Longitudinal Cohort Study. Am J Nephrol 2020;51:975-81. 
18. Beaver CC, Magnan MA. Managing Chemotherapy Side Effects: Achieving Reliable and Equitable Outcomes. Clin J Oncol Nurs 2016;20:589-91.

19. Zamanzadeh V, Valizadeh L, Rahmani A, et al. Cancer survivors' experiences of return to work: A qualitative study. Psychooncology 2018;27:2398-404.

20. Carrera PM, Kantarjian HM, Blinder VS. The financial burden and distress of patients with cancer: Understanding and stepping-up action on the financial toxicity of cancer treatment. CA Cancer J Clin 2018;68:153-65.

21. Dinkel A, Herschbach P. Fear of Progression in Cancer Patients and Survivors. Recent Results Cancer Res 2018;210:13-33.

Cite this article as: Kuang X, Long F, Chen H, Huang Y, He L, Chen 1, Xie 1, Li J, Luo Y, Tao H. Correlation research between fear of disease progression and quality of life in patients with lung cancer. Ann Palliat Med 2022;11(1):35-44. doi: 10.21037/ apm-21-2821
22. Temiz G, Durna Z. Evaluation of Quality of Life and Health Care Needs in Cancer Patients Receiving Chemotherapy. J Cancer Educ 2020;35:796-807.

23. Huang HY, Tsai WC, Chou WY, et al. Quality of life of breast and cervical cancer survivors. BMC Womens Health 2017;17:30.

24. Li XX, Du XW, Song W, et al. Effect of continuous nursing care based on the IKAP theory on the quality of life of patients with chronic obstructive pulmonary disease: A randomized controlled study. Medicine (Baltimore) 2020;99:e19543.

(English Language Editor: B. Draper) 